\title{
FOUR LOGARITHMICALLY COMPLETELY MONOTONIC FUNCTIONS INVOLVING GAMMA FUNCTION
}

\author{
Feng Qi, Da-Wei Niu, Jian CaO, and Shou-Xin Chen
}

\begin{abstract}
In this paper, two classes of functions, involving a parameter and the classical Euler gamma function, and two functions, involving the classical Euler gamma function, are verified to be logarithmically completely monotonic in $\left(-\frac{1}{2}, \infty\right)$ or $(0, \infty)$; some inequalities involving the classical Euler gamma function are deduced and compared with those originating from certain problems of traffic flow, due to J. Wendel and A. Laforgia, and relating to the well known Stirling's formula.
\end{abstract}

\section{Introduction}

Recall $[30,44,48]$ that a function $f$ is said to be completely monotonic on an interval $I$ if $f$ has derivatives of all orders on $I$ and

$$
(-1)^{n} f^{(n)}(x) \geq 0
$$

for $x \in I$ and $n \geq 0$. The set of the completely monotonic functions on $I$ is denoted by $\mathcal{C}[I]$. The well known Bernstein's Theorem [48, p. 161] states that $f \in \mathcal{C}[(0, \infty)]$ if and only if

$$
f(x)=\int_{0}^{\infty} e^{-x s} \mathrm{~d} \mu(s),
$$

where $\mu$ is a nonnegative measure on $[0, \infty)$ such that the integral converges for all $x>0$. This expresses that $f \in \mathcal{C}[(0, \infty)]$ if and only if $f$ is a Laplace transform of the measure $\mu$.

Recall $[8,20,30,33,36,39,40]$ also that a positive function $f$ is called logarithmically completely monotonic on an interval $I$ if $f$ has derivatives of

\footnotetext{
Received October 8, 2006.

2000 Mathematics Subject Classification. Primary 33B15, 26A48, 26A51; Secondary $26 \mathrm{D} 20$.

Key words and phrases. logarithmically completely monotonic function, completely monotonic function, ratio of the gamma functions, Kershaw's inequality, Laforgia's inequality, Stirling's formula, Wendel's inequality.

The first and fourth authors were supported partially by NSF of Henan University, China.

The fourth author was partially supported also by NSF (with Grant Number 2007110004) of the Educational Department of Henan Province, China.
} 
all orders on $I$ and its logarithm $\ln f$ satisfies

$$
(-1)^{k}[\ln f(x)]^{(k)} \geq 0
$$

for all $k \in \mathbb{N}$ on $I$. The set of the logarithmically completely monotonic functions on $I$ is denoted by $\mathcal{L}[I]$. In [9, Theorem 1.1] and $[20,40]$ it is pointed out that the logarithmically completely monotonic functions on $(0, \infty)$ can be characterized as the infinitely divisible completely monotonic functions studied by Horn in [21, Theorem 4.4].

It was proved in $[9,30,39,40,44]$ that

$$
\mathcal{L}[I] \subset \mathcal{C}[I],
$$

but not conversely. Stimulated by the papers [36, 39], among other things, it was further revealed in [9] that

$$
\mathcal{S} \backslash\{0\} \subset \mathcal{L}[(0, \infty)] \subset \mathcal{C}[(0, \infty)],
$$

where $\mathcal{S}$ denotes the set of Stieltjes transforms.

From the inclusions (4) and (5), it is easy to see that, in order to show some functions, especially the power-exponential functions or the exponential functions, are completely monotonic, maybe it is sufficient and much simpler or easier to prove the stronger statements that they are logarithmically completely monotonic or Stieltjes transforms.

It is worthwhile to note that there have been a lot of literature on completely monotonic functions and logarithmically completely monotonic functions related to the gamma function, psi function or polygamma functions. Although it is not practicable to list all of these papers, we still would like to offer some of them, for example, $[3,4,5,6,7,10,15,17,19,22,28]$ or $[12,13,14,26,29,34,41,42]$ and the references therein, to the readers.

It is well known that the classical Euler's gamma function $\Gamma(x)$ is defined for $x>0$ as

$$
\Gamma(x)=\int_{0}^{\infty} e^{-t} t^{x-1} \mathrm{~d} t .
$$

The logarithmic derivative of $\Gamma(x)$, denoted by

$$
\psi(x)=\frac{\Gamma^{\prime}(x)}{\Gamma(x)},
$$

is called the psi or digamma function, and $\psi^{(i)}(x)$ for $i \in \mathbb{N}$ are known as the polygamma or multigamma functions. These functions play central roles in the theory of special functions and have lots of extensive applications in many branches, for example, statistics, physics, engineering, and other mathematical sciences.

The Kershaw's inequality in [23] states that the double inequality

$$
\left(x+\frac{s}{2}\right)^{1-s}<\frac{\Gamma(x+1)}{\Gamma(x+s)}<\left(x-\frac{1}{2}+\sqrt{s+\frac{1}{4}}\right)^{1-s}
$$


holds for $0<s<1$ and $x \geq 1$. If taking $s=\frac{1}{2}$ in (8), then

$$
\sqrt{x+\frac{1}{4}}<\frac{\Gamma(x+1)}{\Gamma(x+1 / 2)}<\sqrt{x+\frac{\sqrt{3}-1}{2}} .
$$

In [24], the following Laforgia's inequalities were established: If $0<\lambda<1$ or $\lambda>2$ and $x \geq 0$, then

$$
\frac{\Gamma(x+1)}{\Gamma(x+\lambda)}>\left(x+\frac{\lambda}{2}\right)^{1-\lambda}
$$

which extends the left hand side inequality in (8); If $1<\lambda<2$ and $x \geq 0$, inequality (10) is reversed; If $0<\lambda<1$ and $x \geq 1$, then

$$
\frac{\Gamma(x+1)}{\Gamma(x+\lambda)}<\left(x+\frac{2 \lambda}{3}\right)^{1-\lambda}
$$

If $1<\lambda<2$ and $x \geq 0$, then

$$
\frac{\Gamma(x+1)}{\Gamma(x+\lambda)}>\left(x+\frac{1}{8}+\frac{\lambda}{2}\right)^{1-\lambda}
$$

If $1<\lambda<2$ and $x \geq 1$, then

$$
\frac{\Gamma(x+1)}{\Gamma(x+\lambda)}>\left(x+\frac{1}{10}+\frac{\lambda}{2}\right)^{1-\lambda} .
$$

In particular, setting $\lambda=\frac{1}{2}$ in (11) yields for $x \geq 1$

$$
\frac{\Gamma(x+1)}{\Gamma(x+1 / 2)}<\sqrt{x+\frac{1}{3}} .
$$

It is easy to see that inequality (11) and the right hand side inequality in (8) are not included each other and it is also clear that inequality (14) is better than the right hand side inequality in (9).

Let $s$ and $t$ be two real numbers and $\alpha=\min \{s, t\}$. For $x \in(-\alpha, \infty)$, define

$$
z_{s, t}(x)= \begin{cases}{\left[\frac{\Gamma(x+t)}{\Gamma(x+s)}\right]^{1 /(t-s)}-x,} & s \neq t, \\ e^{\psi(x+s)}-x, & s=t .\end{cases}
$$

In order to establish the best bounds in Kershaw's inequality (8), among other things, the papers $[11,18,38]$ established the following monotonicity and convexity property of $z_{s, t}(x)$ : The function $z_{s, t}(x)$ is either convex and decreasing for $|t-s|<1$ or concave and increasing for $|t-s|>1$.

In [16, p. 123] and [25], while ones studied certain problems of traffic flow, the following double inequality was obtained for $n \in \mathbb{N}$ :

$$
2 \Gamma\left(n+\frac{1}{2}\right) \leq \Gamma\left(\frac{1}{2}\right) \Gamma(n+1) \leq 2^{n} \Gamma\left(n+\frac{1}{2}\right),
$$


which can be rearranged for $n>1$ as

$$
1 \leq\left[\frac{\Gamma(1 / 2) \Gamma(n+1)}{2 \Gamma(n+1 / 2)}\right]^{1 /(n-1)} \leq 2 .
$$

In [43], by using the following double inequality due to J. Wendel in [47]:

$$
\left(\frac{x}{x+a}\right)^{1-a} \leq \frac{\Gamma(x+a)}{x^{a} \Gamma(x)} \leq 1
$$

for $0<a<1$ and $x>0$, inequality (16) was extended and refined as

$$
\sqrt{x} \leq \frac{\Gamma(x+1)}{\Gamma(x+1 / 2)} \leq \sqrt{x+\frac{1}{2}}
$$

for $x>0$.

It is clear that the double inequality (19) is weaker than (9) and the right hand side inequality in (19) is also weaker than (14).

In [10], the following conclusions were established:

(1) The function

$$
f(x)=\frac{\Gamma(x+1)}{(x+c)^{1 / 2} \Gamma(x+1 / 2)}
$$

for $x>\max \left\{-\frac{1}{2},-c\right\}$ is completely monotonic on $(-c, \infty)$ if $c \leq \frac{1}{4}$; the function $\frac{1}{f(x)}$ is completely monotonic on $\left[-\frac{1}{2}, \infty\right)$ if $c \geq \frac{1}{2}$.

(2) Let $a+1 \geq b>a, \alpha=\max \{-a,-c\}, \beta=\max \{-b,-c\}$ and

$$
g(x ; a, b, c)=(x+c)^{a-b} \frac{\Gamma(x+b)}{\Gamma(x+a)}
$$

for $x>\alpha$. Then the function $g(x ; a, b, c)$ is completely monotonic on $(\alpha, \infty)$ if $c \leq \frac{a+b-1}{2}$ and the function $\frac{1}{g(x ; a, b, c)}$ is completely monotonic for $x>\beta$ if $c \geq a$.

The middle term in (17) hints us to define

$$
g(x)= \begin{cases}{\left[\frac{\Gamma(1 / 2) \Gamma(x+1)}{2 \Gamma(x+1 / 2)}\right]^{1 /(x-1)},} & x \neq 1 \\ \exp \left[1-\gamma-\psi\left(\frac{3}{2}\right)\right], & x=1\end{cases}
$$

for $x \in\left(-\frac{1}{2}, \infty\right)$, where $\gamma=0.57721566 \cdots$ is the Euler-Mascheroni constant, and to consider its logarithmically complete monotonicity.

The first main result of this paper is the following:

Theorem 1.1. $g(x) \in \mathcal{L}\left[\left(-\frac{1}{2}, \infty\right)\right]$ with

$$
\lim _{x \rightarrow-\frac{1}{2}+} g(x)=\infty \quad \text { and } \quad \lim _{x \rightarrow \infty} g(x)=1 .
$$


Remark 1. From the decreasingly monotonic property of $g(x)$,

$$
\lim _{x \rightarrow \infty} g(x)=1 \quad \text { and } \quad g(1)=\exp \left[1-\gamma-\psi\left(\frac{3}{2}\right)\right],
$$

it is obtained that

$$
\begin{aligned}
2 \Gamma\left(x+\frac{1}{2}\right) & \leq \Gamma\left(\frac{1}{2}\right) \Gamma(x+1) \\
& \leq 2 \Gamma\left(x+\frac{1}{2}\right) \exp \left\{(x-1)\left[1-\gamma-\psi\left(\frac{3}{2}\right)\right]\right\}
\end{aligned}
$$

for $x \in[1, \infty)$. From $g(0)=2, \lim _{x \rightarrow \infty} g(x)=1$ and the decreasingly monotonicity of $g(x)$, it is also revealed that

$$
2 \Gamma\left(x+\frac{1}{2}\right) \leq \Gamma\left(\frac{1}{2}\right) \Gamma(x+1) \leq 2^{x} \Gamma\left(x+\frac{1}{2}\right)
$$

for $x \in(0, \infty)$.

Inequalities (24) and (25) extend (16) and (17) and the right hand side inequality of (24) refines the right hand side inequality of (16) and (17). Therefore, it can be said that Theorem 1.1 generalizes, extends, and refines the double inequalities (16) and (17).

Remark 2. Numerical calculation shows

$$
\sqrt{1+\frac{1}{3}}=1.15 \cdots>\frac{2}{\Gamma(1 / 2)} \exp \left\{(1-1)\left[1-\gamma-\psi\left(\frac{3}{2}\right)\right]\right\}=1.12 \cdots
$$

and

$$
\sqrt{2+\frac{1}{3}}=1.5 \cdots<\frac{2}{\Gamma(1 / 2)} \exp \left\{(2-1)\left[1-\gamma-\psi\left(\frac{3}{2}\right)\right]\right\}=1.6 \cdots,
$$

hence, inequality of (14) and the following inequality

$$
\frac{\Gamma(x+1)}{\Gamma(x+1 / 2)} \leq \frac{2}{\Gamma(1 / 2)} \exp \left\{(x-1)\left[1-\gamma-\psi\left(\frac{3}{2}\right)\right]\right\}
$$

for $x \in[1, \infty)$, which is rearrangement of the right hand side inequality of (24), are not included each other. Similarly, it is easy to show that inequality (26) and the right hand side inequality in (19) are also not included each other for $x \in[1, \infty)$.

The left hand side inequality in (18) reminds us to introduce

$$
h_{a}(x)=\frac{(x+a)^{1-a} \Gamma(x+a)}{x \Gamma(x)}
$$

for $x>0$ and $a>0$ and to discuss its logarithmically complete monotonicity.

The second main result of this paper is the following:

Theorem 1.2. (1) $h_{a}(x) \in \mathcal{L}[(0, \infty)]$ if $0<a<1$.

(2) $\left[h_{a}(x)\right]^{-1} \in \mathcal{L}[(0, \infty)]$ if $a>1$. 
(3) For any $a>0$,

$$
\lim _{x \rightarrow 0+} h_{a}(x)=\frac{\Gamma(a+1)}{a^{a}} \quad \text { and } \quad \lim _{x \rightarrow \infty} h_{a}(x)=1 .
$$

Remark 3. It is easy to see that Theorem 1.2 is not included in [10, Theorem 1 and Theorem 3] mentioned above, the complete monotonicities of the functions $f(x)$ and $g(x ; a, b, c)$ defined by $(20)$ and $(21)$.

Remark 4. Since

$$
h_{a}(1)=(1+a)^{1-a} \Gamma(1+a)
$$

and $h_{a}(x)$ is monotonic in $[1, \infty)$ for given $a>0$ and $a \neq 1$, it is deduced from Theorem 1.2 that

$$
\left(\frac{x+a}{1+a}\right)^{1-a} \frac{1}{\Gamma(1+a)} \leq \frac{\Gamma(x+1)}{\Gamma(x+a)}<(x+a)^{1-a}
$$

in $[1, \infty)$ for $0<a<1$. Especially, for $x \in[1, \infty)$,

$$
\sqrt{\frac{\pi}{6}}\left(x+\frac{1}{2}\right)^{1 / 2} \leq \frac{\Gamma(x+1)}{\Gamma(x+1 / 2)}<\left(x+\frac{1}{2}\right)^{1 / 2} .
$$

The well known software Mathematica 5.2 shows that the left hand side inequalities in (8) and (29) are not contained each other. So do the left hand side inequalities in (9) and (30).

If $a>1$, inequalities (29) and (30) are reversed. It is easy to see that the range of the parameter $a>1$ in the reversed inequality of (29) is more extensive than that of $1<\lambda<2$ in (12) and (13) although the lower bound in the reversed inequality of (29) under the conditions $x \geq 1$ and $1<a<2$ is not better than those in inequalities (12) and (13).

In order to obtain a refined upper bound in (18), let us consider the logarithmically complete monotonicity of the function

$$
f_{a}(x)=\frac{\Gamma(x+a)}{x^{a} \Gamma(x)}
$$

the middle term in (18), for $x \in(0, \infty)$ and $a \in(0, \infty)$.

The third main result of this paper is the following:

Theorem 1.3. (1) $f_{a}(x) \in \mathcal{L}[(0, \infty)]$ and $\lim _{x \rightarrow 0+} f_{a}(x)=\infty$ if $a>1$.

(2) $\left[f_{a}(x)\right]^{-1} \in \mathcal{L}[(0, \infty)]$ and $\lim _{x \rightarrow 0+} f_{a}(x)=0$ if $0<a<1$.

(3) $\lim _{x \rightarrow \infty} f_{a}(x)=1$ for any $a \in(0, \infty)$.

Remark 5. It is also easy to see that Theorem 1.3 is not also included in $[10$, Theorem 1 and Theorem 3] mentioned above, the complete monotonicities of the functions $f(x)$ and $g(x ; a, b, c)$ defined by (20) and (21).

Remark 6 . From the fact that $f_{a}(1)=\Gamma(1+a)$ and the monotonicity of $f_{a}(x)$, it follows that

$$
x^{1-a}<\frac{\Gamma(x+1)}{\Gamma(x+a)} \leq \frac{x^{1-a}}{\Gamma(1+a)}
$$


in $[1, \infty)$ for $0<a<1$. If $a>1$, then inequality $(32)$ is reversed.

Easily numerical calculation reveals that for $x \in[1, \infty)$ and $0<a<1$ the right hand side inequalities in (32) and (8) and inequality (11) are not included each other. Under the conditions $1<a<2$ and $x \geq 1$ the reversed inequality (32) and inequalities (12) and (13) are not included each other.

As a straightforward consequence of combining Theorem 1.2 and Theorem 1.3, the following refinement of the upper bound in inequality (18) is established.

Theorem 1.4. Let $x \in(0, \infty)$. If $0<a<1$, then

$$
\begin{aligned}
(33)\left(\frac{x}{x+a}\right)^{1-a} & <\frac{\Gamma(x+a)}{x^{a} \Gamma(x)} \\
& < \begin{cases}\frac{\Gamma(a+1)}{a^{a}}\left(\frac{x}{x+a}\right)^{1-a} \leq 1, & 0<x \leq \frac{a p(a)}{1-p(a)} \\
1, & \frac{a p(a)}{1-p(a)}<x<\infty,\end{cases}
\end{aligned}
$$

where

$$
p(x)= \begin{cases}{\left[\frac{x^{x}}{\Gamma(x+1)}\right]^{1 /(1-x)},} & x \neq 1, \\ e^{-\gamma}, & x=1 .\end{cases}
$$

If $a>1$, the reversed inequality of (33) holds.

Remark 7 . The graph of the function $\frac{a p(a)}{1-p(a)}$, pictured by MATHEMATICA 5.2, shows that it is an increasing function from $(0, \infty)$ to $(0, \infty)$.

Finally, as a by-product, the logarithmically complete monotonicity of the function $p(x)$ defined by (34) is presented as follows.

Theorem 1.5. $p(x) \in \mathcal{L}[(0, \infty)]$ with $\lim _{x \rightarrow 0+} p(x)=1$ and $\lim _{x \rightarrow \infty} p(x)=\frac{1}{e}$.

Remark 8. From Theorem 1.5, it is deduced easily that

$$
\frac{x}{e}<[\Gamma(x)]^{1 /(x-1)} \leq \frac{x}{e^{\gamma}}
$$

for $x \in[1, \infty)$, which improved inequality (4.1) in [2, Theorem 2] and inequality (1.6) in [7, Theorem 1.5] partially.

Further, if taking $x=n+1$ in (35), then

$$
e^{-n}(n+1)^{n}<n ! \leq e^{-\gamma n}(n+1)^{n}
$$

holds for $n \in \mathbb{N}$, which is related to the well known Stirling's formula $[1,16$, $27,45,46]$.

Remark 9. For more information on the history and background of this topic, please refer to $[31,32,35,37]$ and the related references therein. 


\section{Proofs of theorems}

It is well-known that, for $x>0$ and $\omega>0$,

$$
\frac{1}{x^{\omega}}=\frac{1}{\Gamma(\omega)} \int_{0}^{\infty} t^{\omega-1} e^{-x t} \mathrm{~d} t
$$

and that, for $k \in \mathbb{N}$ and $x>0$,

$$
\begin{gathered}
\psi(x)=\ln x+\int_{0}^{\infty}\left(\frac{1}{u}-\frac{1}{1-e^{-u}}\right) e^{-x u} \mathrm{~d} u \\
\psi^{(k)}(x)=(-1)^{k+1} \int_{0}^{\infty} \frac{t^{k}}{1-e^{-t}} e^{-x t} \mathrm{~d} t
\end{gathered}
$$

Moreover, as $x \rightarrow \infty$, the following asymptotic formula holds:

$$
x^{b-a} \frac{\Gamma(x+a)}{\Gamma(x+b)}=1+\frac{(a-b)(a+b-1)}{2 x}+O\left(\frac{1}{x}\right)
$$

where $a$ and $b$ are two constants.

It is remaked that formulas (37), (38), (39) and (40) can be found in [1, p. 257 and p. 259] and $[27,30,33,36,38,39,40,45,46]$.

Proof of Theorem 1.1. Taking logarithm of $g(x)$ leads to

$$
\begin{aligned}
\ln g(x) & =\frac{\ln \Gamma(x+1)+\ln \Gamma(1 / 2)-\ln \Gamma(x+1 / 2)-\ln 2}{x-1} \\
& =\frac{\ln \Gamma(x+1)-\ln 2}{x-1}-\frac{\ln \Gamma(x+1 / 2)-\ln \Gamma(1 / 2)}{x-1} \\
& =\frac{\ln \Gamma(x+1)-\ln \Gamma(1+1)}{x-1}-\frac{\ln \Gamma(x+1 / 2)-\ln \Gamma(1+1 / 2)}{x-1} \\
& =\frac{1}{x-1} \int_{1}^{x} \psi(u+1) \mathrm{d} u-\frac{1}{x-1} \int_{1}^{x} \psi\left(u+\frac{1}{2}\right) \mathrm{d} u \\
& =\frac{1}{x-1} \int_{1}^{x}\left[\psi(u+1)-\psi\left(u+\frac{1}{2}\right)\right] \mathrm{d} u \\
& =\frac{1}{x-1} \int_{1}^{x} \int_{1 / 2}^{1} \psi^{\prime}(u+t) \mathrm{d} t \mathrm{~d} u \\
& =\int_{1 / 2}^{1}\left[\frac{1}{x-1} \int_{1}^{x} \psi^{\prime}(u+t) \mathrm{d} u\right] \mathrm{d} t \\
& =\int_{1 / 2}^{1} \int_{0}^{1} \psi^{\prime}((x-1) u+t+1) \mathrm{d} u \mathrm{~d} t
\end{aligned}
$$

and, for $k \in \mathbb{N}$,

$$
(-1)^{k}[\ln g(x)]^{(k)}=\int_{1 / 2}^{1} \int_{0}^{1} u^{k}\left[(-1)^{k} \psi^{(k+1)}((x-1) u+t+1)\right] \mathrm{d} u \mathrm{~d} t \geq 0
$$

by considering formula (39). This means $g(x) \in \mathcal{L}\left[\left(-\frac{1}{2}, \infty\right)\right]$. 
By L'Hospital's rule and formula (38), it is deduced that

$$
\begin{aligned}
& \lim _{x \rightarrow \infty} \ln g(x)=\lim _{x \rightarrow \infty}\left[\psi(x+1)-\psi\left(x+\frac{1}{2}\right)\right] \\
= & \lim _{x \rightarrow \infty} \ln \frac{x+1}{x+1 / 2}+\lim _{x \rightarrow \infty} \int_{0}^{\infty}\left(\frac{1}{u}-\frac{1}{1-e^{-u}}\right)\left(e^{-u}-e^{-u / 2}\right) e^{-x u} \mathrm{~d} u=0,
\end{aligned}
$$

which can be restated as $\lim _{x \rightarrow \infty} g(x)=1$.

It is easy to see that

$$
\lim _{x \rightarrow-\frac{1}{2}+} \ln g(x)=\infty \quad \text { and } \quad \lim _{x \rightarrow-\frac{1}{2}+} g(x)=\infty .
$$

The proof of Theorem 1.1 is complete.

Proof of Theorem 1.2. Using the differences equation $\Gamma(x+1)=x \Gamma(x)$ and taking limit directly gives

$$
\lim _{x \rightarrow 0+} h_{a}(x)=\lim _{x \rightarrow 0} \frac{\Gamma(x+a+1)}{(x+a)^{a} \Gamma(x+1)}=\frac{\Gamma(1+a)}{a^{a}} .
$$

Using the asymptotic expansion (40) yields

$$
h_{a}(x)=\frac{\Gamma(x+a+1)}{(x+a)^{a} \Gamma(x+1)}=\left(1+\frac{a}{x}\right)^{-a}\left[1+\frac{a(a+1)}{2 x}+O\left(\frac{1}{x}\right)\right] \rightarrow 1
$$

as $x \rightarrow \infty$, which means $\lim _{x \rightarrow \infty} h_{a}(x)=1$.

Taking logarithm of $h_{a}(x)$, differentiating with respect to $x$ successively and utilizing formulas (37) and (39) leads to

$$
\ln h_{a}(x)=(1-a) \ln (x+a)+\ln \Gamma(x+a)-\ln \Gamma(x+1)
$$

and, for $n \in \mathbb{N}$ and $n>1$,

$$
\begin{aligned}
& {\left[\ln h_{a}(x)\right]^{(n)} } \\
= & (1-a) \frac{(-1)^{n-1}(n-1) !}{(x+a)^{n}}+\psi^{(n-1)}(x+a)-\psi^{(n-1)}(x+1) \\
= & (-1)^{n}\left[(a-1) \frac{(n-1) !}{(x+a)^{n}}+(-1)^{n} \psi^{(n-1)}(x+a)-(-1)^{n} \psi^{(n-1)}(x+1)\right] \\
= & (-1)^{n}\left\{\int_{0}^{\infty}(a-1) e^{-(x+a) t} t^{n-1} \mathrm{~d} t+\int_{0}^{\infty}\left[e^{-(x+a) t}-e^{-(x+1) t}\right] \frac{t^{n-1}}{1-e^{-t}} \mathrm{~d} t\right\} \\
= & (-1)^{n}\left\{\int_{0}^{\infty} \frac{e^{-(x+a) t} t^{n-1}}{1-e^{-t}}\left[(a-1)\left(1-e^{-t}\right)+1-e^{(a-1) t}\right] \mathrm{d} t\right\} \\
\triangleq & (-1)^{n} \int_{0}^{\infty} \frac{e^{-(x+a) t} t^{n-1}}{1-e^{-t}} r(t) \mathrm{d} t
\end{aligned}
$$

It is clear that

$$
r^{\prime}(t)=(a-1) e^{-t}\left(1-e^{a t}\right)
$$


and $r(0)=0$. Therefore, the function $r(t)$ is non-positive for $a>1$ and $r(t) \geq 0$ for $0<a<1$. As a result, $(-1)^{n}\left[\ln h_{a}(x)\right]^{(n)} \leq 0$ for $a>1$ and $(-1)^{n}\left[\ln h_{a}(x)\right]^{(n)} \geq 0$ for $0<a<1$.

By formula (38), it is easy to see that

$$
\begin{aligned}
{\left[\ln h_{a}(x)\right]^{\prime} } & =\frac{1-a}{x+a}+\psi(x+a)-\psi(x+1) \\
& =\frac{1-a}{x+a}+\ln \frac{x+a}{x+1}+\int_{0}^{\infty}\left(\frac{1}{t}-\frac{1}{1-e^{-t}}\right)\left(e^{-a t}-e^{-t}\right) e^{-x t} \mathrm{~d} t \\
& \rightarrow 0
\end{aligned}
$$

as $x \rightarrow \infty$. Since $\left[\ln h_{a}(x)\right]^{\prime}$ is decreasing for $a>1$ and increasing for $0<a<1$, then $\left[\ln h_{a}(x)\right]^{\prime} \geq 0$ for $a>1$ and $\left[\ln h_{a}(x)\right]^{\prime} \leq 0$ for $0<a<1$.

Summing up, for any positive integer $k \in \mathbb{N}$, if $a>1$ then $(-1)^{k}\left[\ln h_{a}(x)\right]^{(k)}$ $\leq 0$, if $0<a<1$ then $(-1)^{k}\left[\ln h_{a}(x)\right]^{(k)} \geq 0$. The proof of Theorem 1.2 is complete.

Proof of Theorem 1.3. Applying (40) reveals

$$
f_{a}(x)=\frac{\Gamma(x+a)}{x^{a} \Gamma(x)}=1+\frac{a(a-1)}{2 x}+O\left(\frac{1}{x}\right) \rightarrow 1
$$

as $x \rightarrow \infty$ for $a \in(0, \infty)$.

From

it follows that

$$
f_{a}(x)=\frac{x^{1-a} \Gamma(x+a)}{\Gamma(x+1)},
$$

$$
\lim _{x \rightarrow 0+} f_{a}(x)= \begin{cases}\infty, & a>1, \\ 0, & 0<a<1 .\end{cases}
$$

Taking logarithm of $f_{a}(x)$ and differentiating yields

$$
\ln f_{a}(x)=\ln \Gamma(x+a)-a \ln x-\ln \Gamma(x)
$$

and, by (37) and (39) for $n>1$,

$$
\begin{aligned}
(-1)^{n}\left[\ln f_{a}(x)\right]^{(n)} & =(-1)^{n}\left[\psi^{(n-1)}(x+a)-\psi^{(n-1)}(x)-a \frac{(-1)^{n-1}(n-1) !}{x^{n}}\right] \\
& =\int_{0}^{\infty}\left(e^{-(x+a) t}-e^{-x t}\right) \frac{t^{n-1}}{1-e^{-t}} \mathrm{~d} t+\int_{0}^{\infty} a e^{-x t} t^{n-1} \mathrm{~d} t \\
& =\int_{0}^{\infty} \frac{t^{n-1}}{1-e^{-t}}\left[e^{-a t}-1+a\left(1-e^{-t}\right)\right] e^{-x t} \mathrm{~d} t \\
& \triangleq \int_{0}^{\infty} \frac{t^{n-1}}{1-e^{-t}} s(t) e^{-x t} \mathrm{~d} t .
\end{aligned}
$$

It is clear that $s(0)=0$ and

$$
s^{\prime}(t)=a\left[1-e^{(1-a) t}\right] e^{-t} .
$$


Thus, standard argument gives

$$
s(t) \begin{cases}\geq 0, & a>1 \\ \leq 0, & 0<a<1\end{cases}
$$

This implies

Since

$$
(-1)^{n}\left[\ln f_{a}(x)\right]^{(n)} \begin{cases}\geq 0, & a>1 \\ \leq 0, & 0<a<1\end{cases}
$$

$$
\begin{aligned}
{\left[\ln f_{a}(x)\right]^{\prime} } & =\psi(x+a)-\psi(x)-\frac{a}{x} \\
& =\int_{0}^{\infty}\left(\frac{1}{t}-\frac{1}{1-e^{-t}}\right)\left(e^{-a t}-1\right) e^{-x t} \mathrm{~d} t+\ln \left(1+\frac{a}{x}\right)-\frac{a}{x} \rightarrow 0
\end{aligned}
$$

as $x \rightarrow \infty$ and the function $\left[\ln f_{a}(x)\right]^{\prime}$ is increasing for $a>1$ and decreasing for $0<a<1$, then

$$
\left[\ln f_{a}(x)\right]^{\prime} \begin{cases}\leq 0, & a>1, \\ \geq 0, & 0<a<1 .\end{cases}
$$

In a word, for $k \in \mathbb{N}$, it follows that

$$
(-1)^{k}\left[\ln f_{a}(x)\right]^{(k)} \begin{cases}\geq 0, & a>1 \\ \leq 0, & 0<a<1 .\end{cases}
$$

The proof of Theorem 1.3 is complete.

Proof of Theorem 1.4. As a direct consequence of Theorem 1.2, a double inequality is obtained: For $0<a<1$ and $x>0$,

$$
\left(\frac{x}{x+a}\right)^{1-a}<\frac{\Gamma(x+a)}{x^{a} \Gamma(x)}<\frac{\Gamma(a+1)}{a^{a}}\left(\frac{x}{x+a}\right)^{1-a} .
$$

For $a>1$ and $x>0$, the double inequality (41) is reversed.

As an easy consequence of Theorem 1.3, an inequality is deduced: For $0<$ $a<1$, inequality

$$
\frac{\Gamma(x+a)}{x^{a} \Gamma(x)}<1
$$

is valid in $x \in(0, \infty)$. For $a>1$, inequality (42) reverses.

It is a standard argument that

$$
\frac{\Gamma(a+1)}{a^{a}}\left(\frac{x}{x+a}\right)^{1-a} \leq 1
$$

if and only if

$$
0<x \leq \frac{a p(a)}{1-p(a)}
$$

for $0<a<1$. The proof of Theorem 1.4 is complete. 
Proof of Theorem 1.5. From the differences equation $\Gamma(x+1)=x \Gamma(x)$, it follows easily that

$$
\psi(x+1)-\psi(x)=\frac{1}{x}
$$

for $x>0$. Taking logarithm of $p(x)$ and utilizing (43) gives

$$
\begin{aligned}
\ln p(x) & =\frac{x \ln x-\ln \Gamma(x+1)}{1-x} \\
& =\frac{\ln \Gamma(x+1)-\ln \Gamma(1+1)}{x-1}-\frac{x \ln x-1 \ln 1}{x-1} \\
& =\frac{1}{x-1} \int_{1}^{x} \psi(u+1) \mathrm{d} u-\frac{1}{x-1} \int_{1}^{x}(1+\ln u) \mathrm{d} u \\
& =\frac{1}{x-1} \int_{1}^{x}[\psi(u+1)-\ln u] \mathrm{d} u-1 \\
& =\frac{1}{x-1} \int_{1}^{x}\left[\psi(u)-\ln u+\frac{1}{u}\right] \mathrm{d} u-1 \\
& \triangleq \frac{1}{x-1} \int_{1}^{x} \Psi(u) \mathrm{d} u-1 \\
& =\int_{0}^{1} \Psi((x-1) u+1) \mathrm{d} u-1 .
\end{aligned}
$$

Formulas (37) and (38) imply that

$$
\Psi(x)=\psi(x)-\ln x+\frac{1}{x}=\int_{0}^{\infty}\left[\frac{e^{u}-1-u}{u\left(e^{u}-1\right)}\right] e^{-x u} \mathrm{~d} u .
$$

Since $e^{u}-1-u>0$ for $u \in(0, \infty)$, then $\Psi(x) \in \mathcal{C}[(0, \infty)]$. Therefore, for $k \in \mathbb{N}$,

$$
(-1)^{k}[\ln p(x)]^{(k)}=\int_{0}^{1} u^{k}\left[(-1)^{k} \Psi^{(k)}((x-1) u+1)\right] \mathrm{d} u \geq 0 .
$$

This means $p(x) \in \mathcal{L}[(0, \infty)]$.

The L'Hôspital's rule and formulas (38) and (43) yield

$$
\begin{aligned}
\lim _{x \rightarrow \infty} \ln p(x) & =\lim _{x \rightarrow \infty} \frac{x \ln x-\ln \Gamma(x+1)}{1-x} \\
& =\lim _{x \rightarrow \infty}[\psi(x+1)-(1+\ln x)] \\
& =\lim _{x \rightarrow \infty}\left[\psi(x)-\ln x+\frac{1}{x}\right]-1 \\
& =\lim _{x \rightarrow \infty}[\psi(x)-\ln x]+\lim _{x \rightarrow \infty} \frac{1}{x}-1 \\
& =-1 .
\end{aligned}
$$

Thus, it follows easily that $\lim _{x \rightarrow \infty} p(x)=\frac{1}{e}$. 
It is clear that $\lim _{x \rightarrow 0+} \ln p(x)=0$. Hence, the $\operatorname{limit}_{x \rightarrow 0+} p(x)=1$ follows. The proof of Theorem 1.5 is complete.

Acknowledgements. The authors would like to heartily express many thanks to the anonymous referees for their crucial comments on the original version of this paper.

\section{References}

[1] M. Abramowitz and I. A. Stegun, Handbook of Mathematical Functions, with Formulas, Graphs, and Mathematical Tables, National Bureau of Standards Applied Mathematics Series, 55 Superintendent of Documents, U.S. Government Printing Office, Washington, D.C., 1965.

[2] H. Alzer, Inequalities for the gamma function, Proc. Amer. Math. Soc. 128 (2000), no. $1,141-147$.

[3] __ On some inequalities for the gamma and psi functions, Math. Comp. 66 (1997), no. $217,373-389$.

[4] _ Sharp inequalities for the digamma and polygamma functions, Forum Math. 16 (2004), no. 2, 181-221.

[5] _ Some gamma function inequalities, Math. Comp. 60 (1993), no. 201, 337-346.

[6] H. Alzer and C. Berg, Some classes of completely monotonic functions, Ann. Acad. Sci. Fenn. Math. 27 (2002), no. 2, 445-460.

[7] G. D. Anderson and S.-L. Qiu, A monotoneity property of the gamma function, Proc. Amer. Math. Soc. 125 (1997), no. 11, 3355-3362.

[8] R. D. Atanassov and U. V. Tsoukrovski, Some properties of a class of logarithmically completely monotonic functions, C. R. Acad. Bulgare Sci. 41 (1988), no. 2, 21-23.

[9] C. Berg, Integral representation of some functions related to the gamma function, Mediterr. J. Math. 1 (2004), no. 4, 433-439.

[10] J. Bustoz and M. E. H. Ismail, On gamma function inequalities, Math. Comp. 47 (1986), no. 176, 659-667.

[11] Ch.-P. Chen, Monotonicity and convexity for the gamma function, J. Inequal. Pure Appl. Math. 6 (2005), no. 4, Art. 100; Available online at http://jipam.vu.edu.au/ article $\cdot$ php?sid=574.

[12] Ch.-P. Chen and F. Qi, Logarithmically completely monotonic functions relating to the gamma function, J. Math. Anal. Appl. 321 (2006), no. 1, 405-411.

[13] _ L Logarithmically complete monotonicity properties for the gamma functions, Aust. J. Math. Anal. Appl. 2 (2005), no. 2, Art. 8; Available online at http://ajmaa. org/cgi-bin/paper.pl?string=v2n2/V2I2P8, tex.

[14] Logarithmically completely monotonic ratios of mean values and an application, Glob. J. Math. Math. Sci. 1 (2005), no. 1, 71-76. RGMIA Res. Rep. Coll. 8 (2005), no. 1, Art. 18, 147-152; Available online at http://rgmia.vu.edu.au/v8n1.html.

[15] W. E. Clark and M. E. H. Ismail, Inequalities involving gamma and psi functions, Anal. Appl. (Singap.) 1 (2003), no. 1, 129-140.

[16] M. J. Cloud and B. C. Drachman, Inequalities with Applications to Engineering, Springer Verlag, 1998.

[17] Á. Elbert and A. Laforgia, On some properties of the gamma function, Proc. Amer. Math. Soc. 128 (2000), no. 9, 2667-2673.

[18] N. Elezović, C. Giordano, and J. Pečarić, The best bounds in Gautschi's inequality, Math. Inequal. Appl. 3 (2000), no. 2, 239-252.

[19] A. M. Fink, Kolmogorov-Landau inequalities for monotone functions, J. Math. Anal. Appl. 90 (1982), no. 1, 251-258. 
[20] A. Z. Grinshpan and M. E. H. Ismail, Completely monotonic functions involving the gamma and q-gamma functions, Proc. Amer. Math. Soc. 134 (2006), no. 4, 1153-1160.

[21] R. A. Horn, On infinitely divisible matrices, kernels, and functions, Z. Wahrscheinlichkeitstheorie und Verw. Gebiete 8 (1967), 219-230.

[22] M. E. H. Ismail, L. Lorch, and M. E. Muldoon, Completely monotonic functions associated with the gamma function and its q-analogues, J. Math. Anal. Appl. 116 (1986), no. $1,1-9$.

[23] D. Kershaw, Some extensions of W. Gautschi's inequalities for the gamma function, Math. Comp. 41 (1983), no. 164, 607-611.

[24] A. Laforgia, Further inequalities for the gamma function, Math. Comp. 42 (1984), no. $166,597-600$.

[25] J. Lew, J. Frauenthal, and N. Keyfitz, On the average distances in a circular disc, SIAM Rev. 20 (1978), no. 3, 584-592.

[26] A.-J. Li, W.-Zh. Zhao, and Ch.-P. Chen, Logarithmically complete monotonicity and Shur-convexity for some ratios of gamma functions, Univ. Beograd. Publ. Elektrotehn. Fak. Ser. Mat. 17 (2006), 88-92.

[27] W. Magnus, F. Oberhettinger, and R. P. Soni, Formulas and Theorems for the Special Functions of Mathematical Physics, Third enlarged edition. Die Grundlehren der mathematischen Wissenschaften, Band 52 Springer-Verlag New York, Inc., New York 1966.

[28] M. E. Muldoon, Some monotonicity properties and characterizations of the gamma function, Aequationes Math. 18 (1978), no. 1-2, 54-63.

[29] F. Qi, A class of logarithmically completely monotonic functions and the best bounds in the first Kershaw's double inequality, J. Comput. Appl. Math. 206 (2007), no. 2, 1007-1014.

[30] __ Certain logarithmically $N$-alternating monotonic functions involving gamma and q-gamma functions, Nonlinear Funct. Anal. Appl. 13 (2008), no. 1, in press.

[31] ___ Three classes of logarithmically completely monotonic functions involving gamma and psi functions, Integral Transforms Spec. Funct. 18 (2007), no. 7, 503-509.

[32] F. Qi, J. Cao, and D.-W. Niu, Four logarithmically completely monotonic functions involving gamma function and originating from problems of traffic flow, RGMIA Res. Rep. Coll. 9 (2006), no. 3, Art. 9; Available online at http://rgmia.vu.edu.au/v9n3. html.

[33] F. Qi and Ch.-P. Chen, A complete monotonicity property of the gamma function, J. Math. Anal. Appl. 296 (2004), no. 2, 603-607.

[34] F. Qi, Sh.-X. Chen, and W.-S. Cheung, Logarithmically completely monotonic functions concerning gamma and digamma functions, Integral Transforms Spec. Funct. 18 (2007), no. $6,435-443$.

[35] F. Qi and B.-N. Guo, A class of logarithmically completely monotonic functions and the best bounds in the second Kershaw's double inequality, J. Comput. Appl. Math. 212 (2008), no. 2, 444-456. RGMIA Res. Rep. Coll. 10 (2007), no. 2, Art. 5; Available online at http://rgmia.vu .edu .au/v10n2.html.

[36] _ Complete monotonicities of functions involving the gamma and digamma functions, RGMIA Res. Rep. Coll. 7 (2004), no. 1, Art. 8, 63-72; Available online at http://rgmia.vu.edu.au/v7n1.html.

[37] Wendel-Gautschi-Kershaw's inequalities and sufficient and necessary conditions that a class of functions involving ratio of gamma functions are logarithmically completely monotonic, RGMIA Res. Rep. Coll. 10 (2007), no. 1, Art. 2; Available online at http://rgmia.vu.edu.au/v10n1.html.

[38] F. Qi, B.-N. Guo, and Ch.-P. Chen, The best bounds in Gautschi-Kershaw inequalities, Math. Inequal. Appl. 9 (2006), no. 3, 427-436. 
[39] __ Some completely monotonic functions involving the gamma and polygamma functions, RGMIA Res. Rep. Coll. 7 (2004), no. 1, Art. 5, 31-36; Available online at http://rgmia.vu.edu.au/v7n1.html.

[40] _ - Some completely monotonic functions involving the gamma and polygamma functions, J. Aust. Math. Soc. 80 (2006), no. 1, 81-88.

[41] F. Qi, D.-W. Niu, and J. Cao, Logarithmically completely monotonic functions involving gamma and polygamma functions, J. Math. Anal. Approx. Theory 1 (2006), no. 1, 66-74.

[42] F. Qi, Q. Yang, and W. Li, Two logarithmically completely monotonic functions connected with gamma function, Integral Transforms Spec. Funct. 17 (2006), no. 7, 539-542.

[43] J. Sándor, On certain inequalities for the Gamma function, RGMIA Res. Rep. Coll. 9 (2006), no. 1, Art. 11, 115-117; Ava.ilable online at http://rgmia.vu . edu .au/v9n1 .html.

[44] H. van Haeringen, Completely Monotonic and Related Functions, Report 93-108, Faculty of Technical Mathematics and Informatics, Delft University of Technology, Delft, The Netherlands, 1993.

[45] Zh.-X. Wang and D.-R. Guo, Special Functions, Translated from the Chinese by Guo and X. J. Xia. World Scientific Publishing Co., Inc., Teaneck, NJ, 1989.

[46] __ Tèshā Hánshù Gàilùn, The Series of Advanced Physics of Peking University, Peking University Press, Beijing, China, 2000. (Chinese)

[47] J. G. Wendel, Note on the gamma function, Amer. Math. Monthly 55 (1948), 563-564.

[48] D. V. Widder, The Laplace Transform, Princeton Mathematical Series, v. 6. Princeton University Press, Princeton, N. J., 1941.

\section{FENG Q1}

College of Mathematios and Information Science

HENAN UNIVERSITY

Kaifeng City, Henan Province, 475001, China;

Research Institute of Mathematical Inequality Theory

Henan Polytechnic University

Jiaozuo City, Henan Province, 454010, China

$E$-mail address: qifeng618@gmail.com, qifeng618@hotmail.com, qifeng618@qq.com

DA-WEI NIU

College of Information and Business

ZHONGYUAN UNIVERSITY OF TECHNOLOGY

Zhengzhou City, Henan Province, 450007, China

$E$-mail address: nnddww@gamil.com, nnddww@hotmail.com, nnddww@163.com

JIAN CAO

Department of Mathematics

East China Normal University

Shanghai City, 200241, China

E-mail address: 21caojian@163.com, goodfriendforeve@163.com

SHOU-XIN CHEN

College of Mathematics and Information Science

HENAN UNIVERSITY

Kaifeng City, Henan Province, 475001, China

E-mail address: chensx@henu.edu.cn 